\title{
Avaliação da satisfação dos clientes de uma empresa do setor de serviços: através do modelo Kano e da matriz de importância $x$ desempenho
}

Este artigo busca falar sobre fatores da satisfação dos clientes no setor de serviços, usando a empresa Vidraçaria União para isto, sabendo que no cenário competitivo a satisfação é essencial e um diferencial. As Teorias de Kano e matriz de importância X desempenho são abordadas de forma explicativa para melhor compreensão. Foi feita a análise da satisfação dos clientes relacionada aos 13 atributos selecionados. 0 questionário foi respondido por 134 clientes, conforme o cálculo baseado na equação de Mattar. A análise de importância $x$ desempenho pode identificar oportunidades de melhoria, sendo apresentadas em forma de matriz bidimensional dividida em quatro quadrantes que classificando em pontos fracos, pontos fortes, pontos fracos menores e pontos fortes menores. O Modelo Kano compara a relação não linear entre desempenho e satisfação com os determinados atributos, classificando estes em Obrigatórios, Atrativos, Unidimensionais, Neutros e Reversos. Destaca-se pela análise dos dados, que a empresa Vidraçaria União atende as expectativas dos consumidores em boa parte dos atributos, sendo que, dos treze atributos a empresa precisa priorizar melhoria em quatro deles devido à baixa satisfação dos clientes com os mesmos, sendo: Demora na entrega, prontidão dos funcionários, rapidez na medição e acessibilidade com os donos.

\section{Customer satisfaction assessment of a service sector company: through the Kano model and the importance $x$ performance matrix}

\begin{abstract}
This article seeks to talk about customer satisfaction factors in the service sector, using the company Vidraçaria União for this, knowing that in the competitive scenario, satisfaction is essential and a differential. Kano Theories and Matrix of Importance vs. Performance are explained in an explanatory manner for better understanding. Customer satisfaction analysis was performed related to the 13 selected attributes. The questionnaire was answered by 134 clients, according to the calculation based on Mattar's equation. The importance $\mathrm{x}$ performance analysis can identify opportunities for improvement, being presented as a twodimensional matrix divided into four quadrants that classify into weaknesses, strengths, minor weaknesses and minor strengths. The Kano Model compares the nonlinear relationship between performance and satisfaction with the given attributes, classifying them as Required, Attractive, One-dimensional, Neutral, and reverse. It is noteworthy by the analysis of the data, that the company Union meets the expectations of consumers in much of the attributes, and of the thirteen attributes the company needs to prioritize improvement in four of them due to low customer satisfaction with them, as follows: Delays in delivery, employee readiness, quick measurement and accessibility with owners.
\end{abstract}

Keywords: Clients satisfaction; Kano model; Importance Matrix vs. Performance.

Topic: Qualidade

Reviewed anonymously in the process of blind peer.
Received: $15 / 10 / 2019$

Approved: $21 / 12 / 2019$
Alan Barion de Sá (iD)

Universidade Estadual do Oeste do Paraná, Brasil http://lattes.cnpq.br/5427535291852543

http://orcid.org/0000-0001-6311-9672

alanbarion@hotmail.com

\section{Silvana Anita Walter (iD)}

Universidade Estadual do Oeste do Paraná, Brasil http://lattes.cnpq.br/6055446867536139

http://orcid.org/0000-0003-1684-5465

silvanaanita.walter@gmail.com

Mario Luiz Soares (it)

Universidade Estadual do Oeste do Paraná, Brasil

http://lattes.cnpq.br/1647974839729079

http://orcid.org/0000-0002-0561-8673

mario.soares@unioeste.br
Cinara Kottwitz Manzano Brenzan (1)

Universidade Estadual do Oeste do Paraná, Brasil

http://lattes.cnpq.br/0181164018755940

http://orcid.org/0000-0002-1684-2320

cinaramanzano@yahoo.com.br
Referencing this:

SÁ, A. B.; WALTER, S. A.; SOARES, M. L.; BRENZAN, C. K. M.. Avaliação da satisfação dos clientes de uma empresa do setor de serviços: através do modelo Kano e da matriz de importância $x$ desempenho. Revista Brasileira de Administração Científica, v.10, n.4, p.73-86, 2019. DOI: http://doi.org/10.6008/CBPC2179-684X.2019.004.0005 


\section{INTRODUÇÃO}

O mundo dos negócios com o mercado globalizado e a competitividade fazem com que as melhores empresas se destaquem, um ponto importante é o marketing de relacionamento que é desenvolvido para garantir relações rentáveis e duradouras, uma vez que boas relações significam maior chance de retorno de um cliente (KLEIN, 2003). Grande parte das empresas prestam serviços se preocupando principalmente com os produtos tangíveis e esquecem que a satisfação dos clientes é que define a posição da empresa frente à concorrência. A qualidade no geral é importante para os clientes e é um diferencial para a empresa no ambiente competitivo (BEDDOW et al., 1995).

Atrair e reter clientes é uma tarefa difícil, pois os clientes têm acesso a uma grande diversidade de produtos e serviços (KOTLER et al., 2003). Com isso em mente, entende-se que a satisfação dos clientes é muito importante para uma empresa se manter e crescer no mercado, pois isso influencia na reputação da empresa que reflete na imagem dela. A pesquisa de satisfação de clientes é como um sistema de administração de informações que ouve e interpreta a voz do cliente, através da avaliação do cliente sobre a empresa a partir da visão do próprio cliente (ROSSI et al., 1998).

Diante do cenário competitivo das vidraçarias, que estão no setor de prestação de serviços e que este por sua vez ocupa uma posição extremamente relevante na economia, existe a necessidade de conquistar e manter clientes para sobrevivência dessas organizações (TINOCO et al., 2007). Como a quantidade de vidraçarias tem crescido e a concorrência tem exigido um melhor gerenciamento e atuação dessas empresas, a avaliação da qualidade de serviços feita por um cliente pode se tornar o diferencial para a organização se alinhada às estratégias gerenciais (GIANESI et al., 1994, MARINHO, 2009).

A insatisfação dos clientes traz consigo muitas desvantagens para empresa, uma vez que um cliente insatisfeito pode divulgar uma imagem negativa da organização. Sabendo dessa necessidade de manter boas relações e manter o cliente satisfeito, se tem como questão norteadora deste artigo: Quais as oportunidades de melhoria que irão aumentar o grau de satisfação dos clientes com o serviço prestado pela empresa Vidraçaria União?.

O objetivo principal do trabalho em questão é destacar oportunidades de melhorias em uma vidraçaria, analisando a satisfação dos clientes com relação aos produtos e serviços, para isso foram distribuídos em 13 atributos com o fim de avaliação. Se tem como objetivos específicos: a) Identificar o perfil dos respondentes; b) Analisar a satisfação atual dos clientes com cada atributo; c) Encontrar pontos fortes e fracos e d) sugerir melhorias. $O$ estilo utilizado foi o modelo Kano e matriz de importância $X$ desempenho.

Estruturou-se o referido artigo após a introdução, nas seguintes seções: na segunda, apresenta-se a revisão de literatura sobre a importância da qualidade dos serviços prestados; na terceira, relatam-se os procedimentos metodológicos empregados para o desenvolvimento da pesquisa; na quarta, se fazem a apresentação e a análise dos resultados obtidos; e na quinta, conclui-se e apresentam-se sugestões para pesquisas futuras. 


\section{REVISÃO TEÓRICA}

\section{Satisfação de clientes}

Clientes felizes com a empresa em questão tendem a se tornar 'fiéis' e influenciadores de outros possíveis clientes. Clientes passam suas experiências adiante, sejam elas agradáveis ou desagradáveis, e essas informações podem chegar a milhares de pessoas. Uma vez que o cliente tem todo esse poder, se observa que torná-lo satisfeito por meio da qualidade de serviços é um fator de extrema importância em uma empresa (LAS CASAS, 2012; MOUTELLA, 2004). A satisfação e a lealdade de um cliente podem ser conquistadas e gerarem lucros para a empresa, assim como a insatisfação pode gerar prejuízos além de fortalecer a concorrência (KOTLER, 1998).

É possível avaliar a satisfação dos clientes através de um questionário, que pode ser adaptado conforme a necessidade da empresa. O questionário deve ser aplicado periódica e aleatoriamente abordando possíveis causas de insatisfação (LAS CASAS et al., 1997). A organização deve buscar sempre ter uma vantagem competitiva identificando e catalogando dados, definindo seus melhores atributos que fazem o cliente optar por tal diferencial (CYRNE et al., 2003, citado por CYRNE, 2011).

As empresas devem buscar a satisfação de seus clientes para usar como vantagem competitiva, considerando-a indispensável para a organização no ambiente competitivo (TONTINI, 2003). Para analisar a satisfação dos clientes em relação às suas expectativas com relação uma empresa, pode se utilizar métodos lineares e métodos não lineares. Neste presente estudo foram utilizadas duas metodologias: Modelo Kano (método não linear) e a Matriz de Importância x Desempenho (linear) respectivamente, para analisar a satisfação dos clientes da Vidraçaria União.

\section{Modelo Kano - Método não linear}

O modelo Kano de qualidade atrativa e obrigatória (KANO, 1984) distingue determinados pontos que podem influenciar na satisfação ou não do cliente, são eles: a) atributos obrigatórios: são atributos básicos que podem levar à insatisfação se não estiverem presentes ou forem insuficientes, entretanto, caso estejam presentes e forem suficientes levarão à satisfação do cliente, pois são atributos minimamente esperados pelo consumidor por parte da organização. Como exemplo a limpeza do ambiente. b) atributos unidimensionais: nestes atributos, a satisfação se dá de forma proporcional ao desempenho do atributo, ou seja, quanto melhor o desempenho maior será a satisfação do cliente e vice-versa. Geralmente atributos unidimensionais são exigidos com clareza pelos clientes. c) atributos atrativos: São atributos que despertam o interesse do cliente, podem trazer satisfação do cliente, mas sua falta não leva à insatisfação dele. 0 atendimento destes atributos ocasiona uma satisfação mais que proporcional, os atributos atrativos não são expressos e nem esperados pelo cliente, sendo especiais, mas sem necessidade.

Além dos 3 atributos mencionados anteriormente ainda há os atributos neutros e os reversivos. 0 modelo Kano considera a não linearidade entre desempenho e satisfação, se manifesta como o resultante do desempenho dos atributos em relação à satisfação dos clientes (HUISKONEN et al., 1998). Alguns atributos 
podem trazer mais satisfação que outros quando estão presentes, por outro lado, alguns atributos obrigatórios podem trazer mais insatisfação do que outros quando tiverem desempenho insuficiente ou não estiverem presentes. O modelo original do Modelo Kano não identifica o grau de satisfação dos clientes, ele identifica apenas o percentual de satisfeitos ou insatisfeitos da organização em questão.

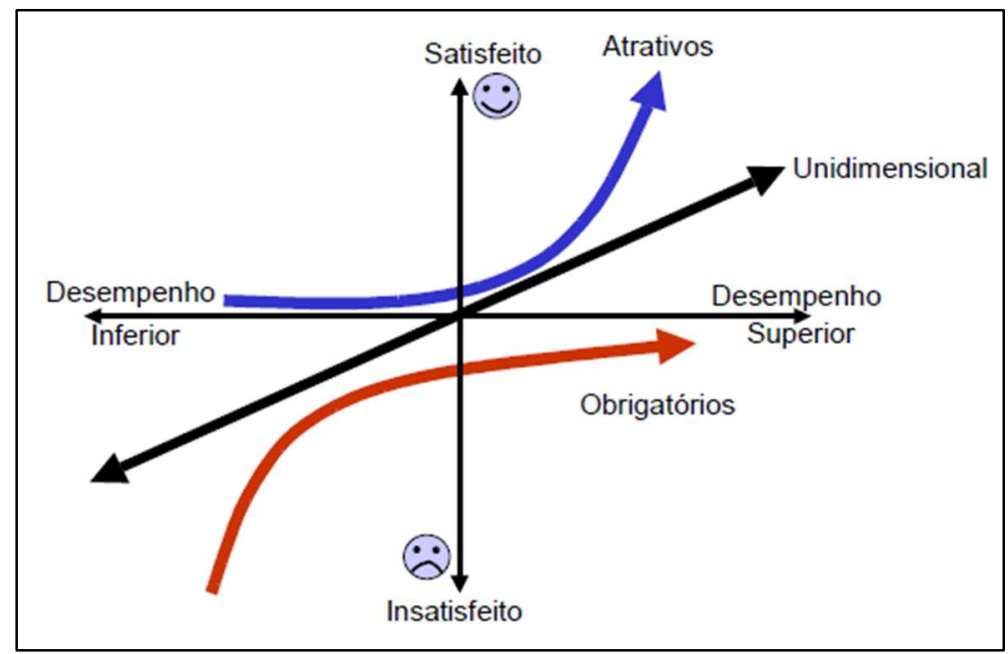

Figura 1: Modelo Kano de qualidade atrativa e obrigatória. Fonte: Adaptado de Matzler et al. (2004).

Tontini (2003) comenta que o gráfico apresenta o desenvolvimento de uma metodologia 'alterada' para identificar atributos no Modelo Kano. Além da modificação do modelo de questionário, Tontini também criou uma maneira modificada de calcular o coeficiente de satisfação dos clientes (BERGER, 1993).

\section{Matriz de Importância x Desempenho - Método Linear}

A análise da Matriz de Importância X Desempenho foi criada por Martilla et al. (1977), ela é um método de análise linear (como o título induz a pensar) que permite que a empresa tenha uma ideia sobre os serviços e produtos que podem ser melhorados para proporcionar satisfação aos seus clientes. Para isso, os clientes avaliam qual é a importância e o desempenho da entidade em questão com relação ao atendimento de suas expectativas para cada atributo da pesquisa. Os dados obtidos são utilizados para elaborar uma matriz bidimensional em que a importância para o cliente sobre o atributo é apresentada pelo eixo y e desempenho da organização é mostrada pelo eixo x, conforme exposto na Figura 2.

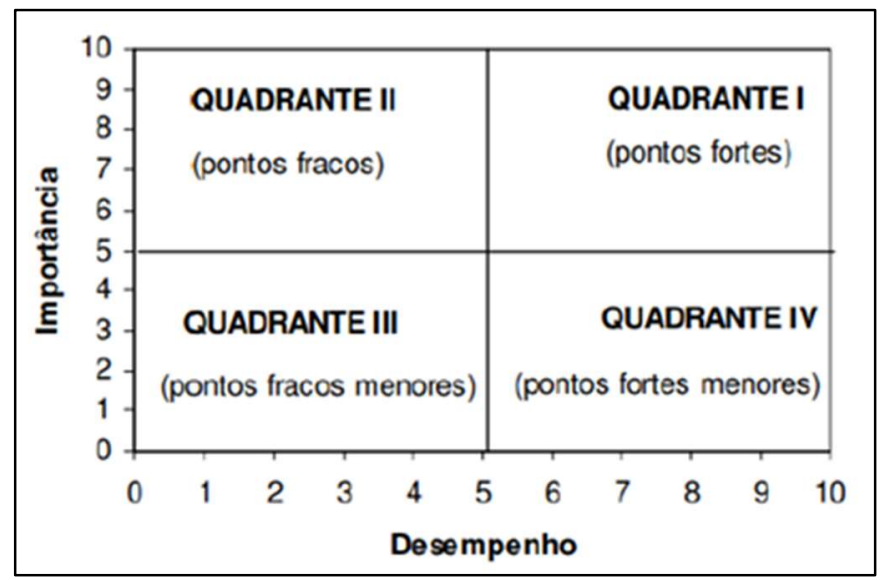

Figura 2: Matriz de Importância x Desempenho. Fonte: Adaptado de Matzler et al. (2004). 
A importância é atribuída, de uma maneira geral, por meio de uma escala direta (Ex.: 1 a 10), sendo relacionado com a satisfação e o desempenho segundo o cliente. $O$ desempenho é atribuído por meio de escala direta (Ex.: 1 a 10), de maneira relativa à concorrência (Ex.: Muito Pior, Pior, Igual, Melhor, Muito Melhor) ou ao desempenho esperado (GARVER, 2003; TONTINI et al., 2004).

A matriz é dividida em quatro quadrantes (Figura 2). Os atributos que se situarem no Quadrante I são considerados pontos fortes devido ao alto desempenho e sua grande importância e, portanto, a representam possível vantagem competitiva. No Quadrante II estão os pontos fracos, que são os atributos de alta importância, porém com baixo desempenho, devem receber mudanças assim que possível. Melhorando os atributos do Quadrante II a empresa melhora com relação à satisfação geral. O Quadrante III possui os atributos de baixa importância e baixo desempenho, a atenção a esses atributos é dispensável, são os pontos fracos menores. Por fim, o Quadrante IV são os atributos de baixa importância com alto desempenho, são os pontos fortes menores.

\section{Estudos empíricos sobre o tema}

Tontini (2005), realizou pesquisa para a identificação de oportunidades de melhoria em Instituições de Ensino Superior (IES), utilizando os mesmos métodos utilizados neste estudo, sendo eles: Matriz de Importância x Desempenho e Modelo Kano de Qualidade Atrativa e Obrigatória. Eles utilizaram 20 atributos do curso de Administração da Universidade Regional de Blumenau/SC para serem analisados através de uma pesquisa quantitativa com uma amostra de 362 alunos de diferentes habilitações, turnos e fases.

Os métodos utilizados para identificação de oportunidades de melhoria foram o Modelo Kano e a Matriz de Importância x Desempenho, explorando-se as diferenças entre os dois. O uso conjunto dos dois permitiu a identificação de atributos prioritários para melhoria com maior facilidade e eficiência. Alguns Atributos que foram considerados como críticos pela Matriz de Importância x Desempenho tiveram sua prioridade reduzida por serem considerados atrativos pelo Modelo Kano. Os resultados demonstraram a potencialidade de aplicação conjunta dos dois métodos como identificadores de oportunidade de melhoria nas IES.

\section{METODOLOGIA}

Para a elaboração desta pesquisa foi utilizado o método quantitativo e o instrumento de coleta de dados foi um questionário com base no Modelo Kano, proposto por Tontini (2003). Os questionários foram aplicados com os clientes da Vidraçaria União no ano de 2019, a fim de obter dados sobre a satisfação deles. Para o cálculo do tamanho da amostra dessa etapa, foi utilizada a equação citada por Mattar (1996), para uma população finita.

$$
n=\frac{N Z^{2} P Q}{\left(E^{2} N+Z^{2} P Q\right)}
$$


Onde:

$n:$ tamanho da amostra;

$N$ : tamanho da população;

$P$ : proporção de ocorrência da variável em estudo na população $(0,5)$;

$Q$ : proporção de não ocorrência da variável em estudo na população $(Q=1-P)$;

$E$ : erro máximo admitido $(0,05)$;

$Z$ : valor padrão determinado pelo nível de confiança. Para o nível de confiança de $95 \%, Z=2$.

$$
\begin{array}{r}
n=200 \cdot 2^{2} \cdot(0,5 \cdot 1-0,5) /\left(0,05^{2} \cdot 200+2^{2}\right) \cdot(0,5 \cdot 1-0,5) \\
n=800 \cdot 0,25 / 0,0025 \cdot 200+4 \cdot 0,25
\end{array}
$$$$
n=200 / 0,5+1
$$

$n=133,333$ ou seja, $n=134$

O resultado do cálculo do tamanho mínimo da amostra foi de 134 questionários, a margem de erro conforme este cálculo é de 5\%. A Vidraçaria União, está voltada para a prestação de serviços e possui 1.225 clientes cadastrados, foram analisados 134 questionários, os quais apresentam 13 atributos da empresa. 0 questionário foi composto por quatro partes. A primeira identificou atributos segundo o modelo Kano. Para tanto foram observadas duas perguntas, descrevendo respectivamente uma situação de aumento e outra de diminuição no desempenho de cada atributo nas quais foram apresentadas aos respondentes. Para cada pergunta o cliente indicou sua expectativa de satisfação ou insatisfação em relação às situações mencionadas, numa escala variando de extremamente insatisfeito a extremamente satisfeito (Quadro 1).

Quadro 1: Questões para identificação dos atributos pelo Modelo Kano.

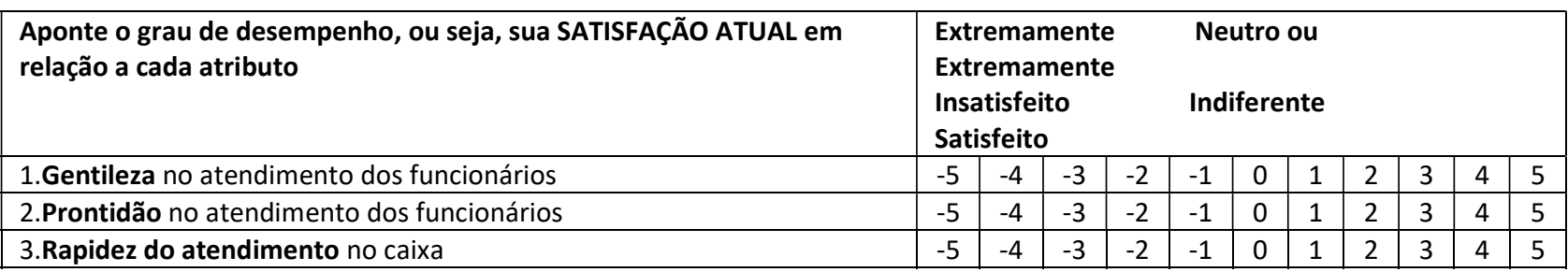

\section{Primeira etapa}

Na segunda etapa mediu-se a satisfação geral atual com a Vidraçaria União e o grau de satisfação existente em relação ao nível de desempenho atual encontrado nos atributos. Novamente a escala variou de -5 (extremamente insatisfeito) a +5 (extremamente satisfeito). Já no terceiro momento verificou-se o grau de importância para cada um dos atributos da empresa, na qual os clientes atribuem notas numa escala de importância de 1 a 5. Na última parte, pesquisou-se dados pessoais e de utilização dos atributos, por meio de perguntas fechadas.

Também foram feitas três perguntas de identificação, referindo-se ao sexo: a) masculino e b) feminino; à faixa etária e estado civil dos respondentes. Em relação à faixa etária. Para complementar o assunto, foram feitas três perguntas, nas quais os clientes indicavam pontos fortes, fracos e sugestões de melhorias.

\section{RESULTADOS E DISCUSSÃO}

Esta sessão apresenta os dados divididos em quatro blocos: Perfil dos respondentes; análise conjunta da Matriz de Importância x Desempenho e Modelo Kano de Qualidade Atrativa e Obrigatória, análise de 
correlação no SPSS e na última etapa, os pontos fortes, fracos e sugestões de melhorias.

\section{Perfil dos Respondentes}

Para iniciar as análises foram caracterizados os respondentes. Duas questões fechadas estão relacionadas ao perfil dos clientes, conforme a Tabela 1.

Tabela 1: Sexo dos respondentes.

\begin{tabular}{|c|c|c|}
\hline Sexo & Quantidade & percentual \\
\hline Feminino & 53 & $39,55 \%$ \\
\hline Masculino & 81 & $60,45 \%$ \\
\hline TOTAL & $\mathbf{1 3 4}$ & $\mathbf{1 0 0 \%}$ \\
\hline
\end{tabular}

Quanto ao gênero se percebe que a maioria dos respondentes é do sexo masculino, correspondendo a $60,45 \%$ do total e o restante $39,55 \%$ do sexo feminino. Em relação à faixa etária, os resultados sobre a idade dos participantes são presentados na Tabela 2.

Tabela 2: Faixa etária dos respondentes.

\begin{tabular}{|c|c|c|c|c|c|}
\hline Faixa etária & Quantidade & Percentual & Faixa etária & Quantidade & Percentual \\
\hline 10 a 20 & 19 & $14,18 \%$ & 51 a 60 & 5 & $3.73 \%$ \\
\hline 21 a 30 & 37 & $27,61 \%$ & 61 a 70 & 10 & $7,46 \%$ \\
\hline 31 a 40 & 40 & $29,85 \%$ & 71 a 80 & 1 & $0,75 \%$ \\
\hline 41 a 50 & 22 & $16,42 \%$ & $81+$ & 0 & $0 \%$ \\
\hline & & & TOTAL & 134 & $100 \%$ \\
\hline
\end{tabular}

Observa-se, por meio da Tabela 2, que há predominância de um público, no qual a maior parte dos respondentes está na faixa etária de 21 a 40 anos, correspondendo a 57,46\% do total, sendo que na faixa acima de 80 anos não houve nenhum respondente. A tabela 3 apresenta o estado civil dos clientes. Nota-se, a partir da Tabela 4, que a maior parte dos respondentes é solteiro(a), representando $58,26 \%$ do total.

Tabela 3: Estado civil.

\begin{tabular}{|l|c|c|}
\hline \multicolumn{1}{|c|}{ Estado civil } & Quantidade & Percentual \\
\hline Solteiro & 64 & $47,76 \%$ \\
\hline Casado & 57 & $42,54 \%$ \\
\hline Viúvo & 7 & $5,22 \%$ \\
\hline Divorciado & 5 & $3,73 \%$ \\
\hline Amasiado & 1 & $0,75 \%$ \\
\hline TOTAL & 134 & $100 \%$ \\
\hline
\end{tabular}

\section{Satisfação Geral}

Em relação à Satisfação Geral, os índices foram contabilizados e os resultados estão apresentados na Tabela 4, na qual a média geral da satisfação foi calculada e os dados apresentaram a seguinte porcentagem.

Tabela 4: Satisfação Geral.

\begin{tabular}{|l|l|}
\hline Satisfação geral & Satisfação geral \\
\hline Média & 3,01 \\
\hline
\end{tabular}

Nota-se pela tabela que a satisfação dos clientes se encontra por volta de 3,01 em uma escala de -5 a +5 , é como $72,90 \%$ de aprovação. Por meio da análise de dados, pode-se dizer que a empresa atinge satisfação mediana em relação a seus serviços. 


\section{Discussões de análise comparativa: matriz de importância x desempenho e modelo Kano}

Os resultados da aplicação dos questionários foram processados procurando-se identificar a classificação dos atributos segundo o Modelo Kano de Qualidade Atrativa e Obrigatória e as oportunidades de melhoria pela Matriz de Importância x Desempenho. Para dividir os quadrantes, foram utilizadas uma das formas de divisão e análise de melhoria proposta por Garver (2003).

\section{Análise pela matriz de importância $\mathrm{x}$ desempenho}

A Figura 3 mostra a Matriz de Importância x Desempenho para os atributos estudados. Um ponto chave na análise pela matriz de Importância x Desempenho é a posição das linhas divisórias dos quadrantes. A matriz da figura 3 pode ser observada de forma ampliada na figura 4 (ZOOM).

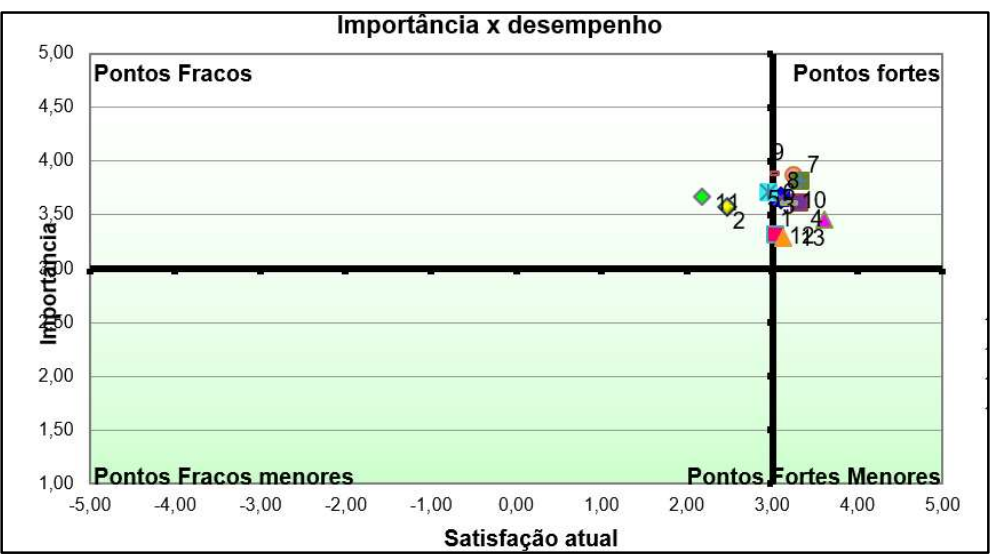

Figura 3: Matriz de Importância x Desempenho para atributos pesquisados. Gráfico Importancia x desempenho.

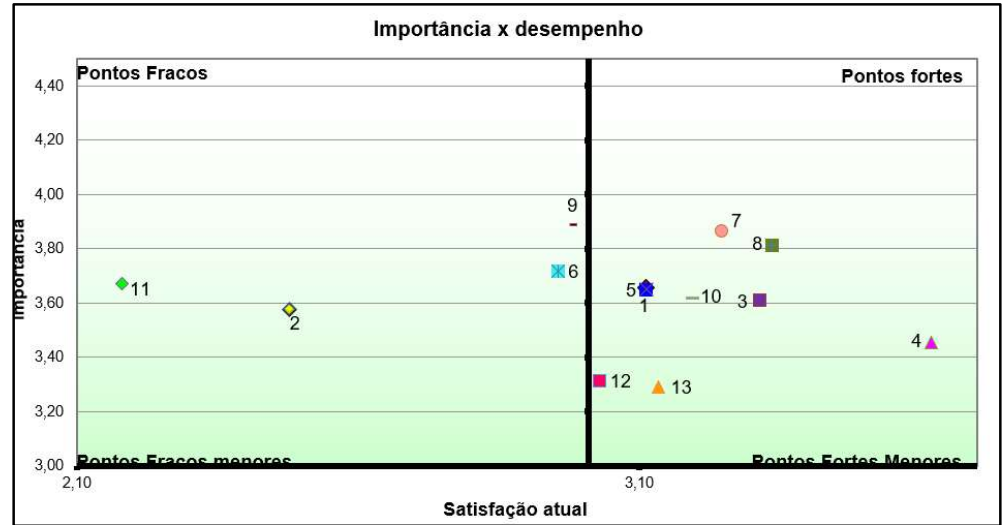

Figura 4: Ampliação da visualização da Matriz de Importância x Desempenho para atributos pesquisados. Legenda:

$\begin{array}{cc}\text { Gentileza no atendimento } & 8 \\ \text { Prontidão dos funcionários } & 9 \\ \text { Rapidez no atendimento } & 10 \\ \text { Apresentação dos funcionários } & 11 \\ \text { Atendimento em domicílio } & 12 \\ \text { Rapidez na medição } & 13 \\ \text { Conservação dos vidros } & -\end{array}$

$$
\begin{gathered}
\text { Variedade de produtos } \\
\text { Acessibilidade com donos } \\
\text { Preços }
\end{gathered}
$$
Demora na entrega Atendimento por telefone Opções de pagamento

Na divisão dos quadrantes foi utilizada uma divisão onde a linha divisória de importância foi estabelecida em Importância (3), e a linha divisória de Satisfação Atual foi estabelecida pela média da Satisfação Geral (Atual) em relação aos atributos (3,01). No Quadrante I, da figura 2 observam-se nove (9) atributos, pontos fortes da pesquisa: Gentileza no atendimento (1); Rapidez no atendimento (3); 
Apresentação dos funcionários (4); Atendimento à domicílio (5); Conservação dos vidros (7); Variedade de produtos (8); Preços (10); Atendimento por telefone (12) e Opções de pagamento (13).

No Quadrante II da figura 2 observam-se quarto (4) atributos, considerados os pontos fracos da pesquisa: Rapidez na medição (6); Acessibilidade com donos (9); demora na entrega (11) e Prontidão dos funcionários (2). No Quadrante III da figura 2 Observa-se como ponto fraco menor a Prontidão dos funcionários (2). No Quadrante IV não há relação de atributos, significa que a empresa não está desperdiçando recursos.

\section{Modelo Kano de qualidade atrativa e obrigatória}

A análise de dados segundo o Modelo Kano encontra-se representada na Figura 5 através do gráfico de dispersão do índice de satisfação do cliente. Neste caso, a linha divisória dos quadrantes foi estabelecida em $+1,00$ para a escala do CS - Coeficiente de Satisfação, e - 1,00 para o $\mathrm{Cl}$ - Coeficiente de Insatisfação. A Figura 5 pode ser visualizada de forma ampliada (zoom) por meio da Figura 6.

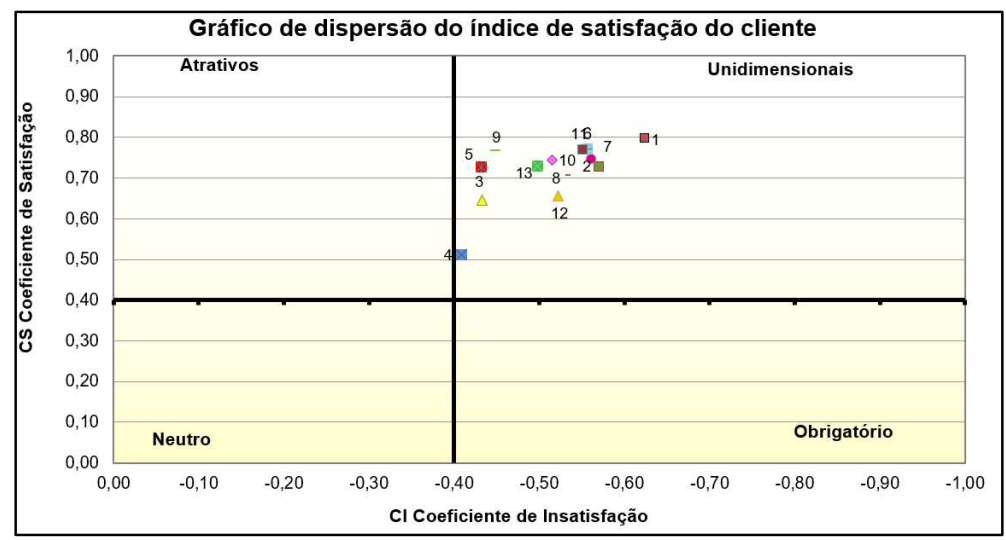

Figura 5: Gráfico de dispersão do índice de satisfação do cliente.

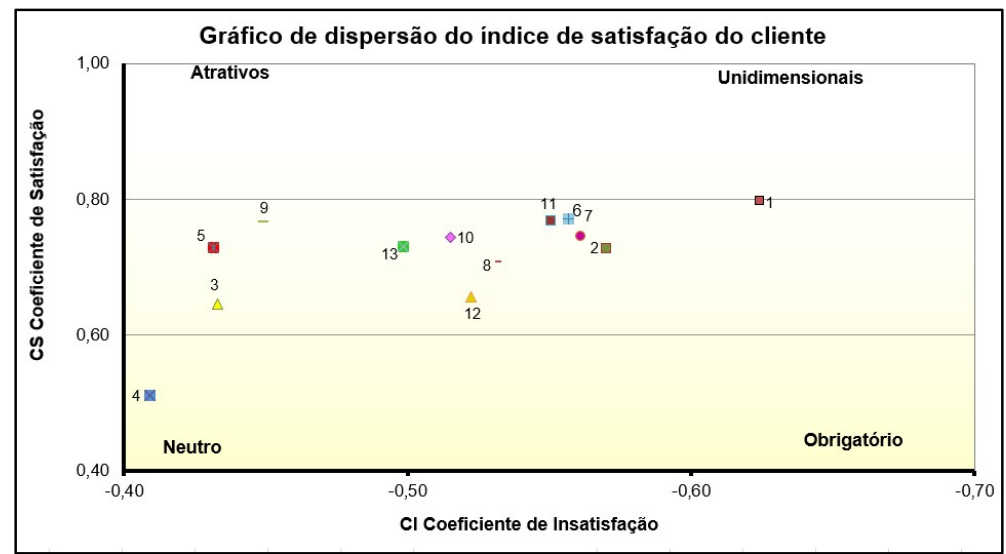

Figura 6: Ampliação da visualização do gráfico de dispersão do índice de satisfação do cliente. Legenda:

$\begin{array}{ccc}\text { Gentileza no atendimento } & 8 & \text { Variedade de produtos } \\ \text { Prontidão dos funcionários } & 9 & \text { Acessibilidade com donos } \\ \text { Rapidez no atendimento } & 10 & \text { Preços } \\ \text { Apresentação dos funcionários } & 11 & \text { Demora na entrega } \\ \text { Atendimento em domicílio } & 12 & \text { Atendimento por telefone } \\ \text { Rapidez na medição } & 13 & \text { Opções de pagamento }\end{array}$

Por meio da figura 6 - "Ampliação da visualização do gráfico de dispersão do índice de satisfação do cliente" - Observa-se que os 13 atributos relacionados apresentam características de atributos 
considerados Unidimensionais, em outras palavras, significa que a satisfação do cliente é proporcional ao nível de desempenho dos atributos. Como todos foram considerados unidimensionais, não houve incidência de atributos no quadrante de obrigatório, neutro e atrativos.

\section{Decisões de melhoria: análise conjunta da matriz de importância $x$ desempenho e modelo Kano de qualidade atrativa e obrigatória}

O Modelo Kano introduz questionamentos sobre o uso da análise da Matriz de Importância $x$ Desempenho para a identificação de oportunidades de melhoria de produtos e serviços. A importância de um atributo varia conforme sua classificação pelo Modelo Kano. O Quadro 2 mostra alguns problemas e decisões errôneas que podem ser tomadas quando não considerada a classificação dos atributos dentro do Modelo Kano.

\section{Modelo Kano x Erros na análise de Importância x Desempenho}

O Modelo Kano introduz questionamentos sobre o uso da análise da Matriz de Importância $x$ Desempenho para a identificação de oportunidades de melhoria de produtos e serviços. A importância de um atributo varia conforme sua classificação pelo Modelo Kano. O Quadro 2 mostra alguns problemas e decisões errôneas que podem ser tomadas quando não considerada a classificação dos atributos dentro do Modelo Kano.

Quadro 2: Modelo Kano x Erros na análise de Importância x Desempenho.

\begin{tabular}{|c|c|c|c|c|c|c|}
\hline Item & Modelo Kano & Importância & $\begin{array}{c}\text { Desempenho } \\
\text { competitivo }\end{array}$ & $\begin{array}{l}\text { Decisão } \\
\text { tomada }\end{array}$ & Razões do erro & Resultado \\
\hline a) & Obrigatório & Alta & Igual & Melhorar & $\begin{array}{l}\text { Melhoria de atributos } \\
\text { obrigatório } \\
\text { acima deste nível não } \\
\text { aumenta a satisfação. }\end{array}$ & $\begin{array}{l}\text { Desperdício de } \\
\text { recursos. }\end{array}$ \\
\hline b) & Obrigatório & Baixa & Superior & Abandonar & $\begin{array}{l}\text { Experiências anteriores } \\
\text { gratificantes e } \\
\text { desempenho } \\
\text { superior levam o } \\
\text { consumidor a classificar } \\
\text { atributo. } \\
\end{array}$ & $\begin{array}{l}\text { Desatenção a um } \\
\text { nível de serviço } \\
\text { necessário, } \\
\text { podendo levar à } \\
\text { insatisfação. }\end{array}$ \\
\hline c) & Obrigatório & Alta & Inferior & Melhorar & - & Decisão corretora. \\
\hline d) & Atrativo & Baixa & Inferior/igual & Abandonar & $\begin{array}{l}\text { Consumidores sem } \\
\text { experiência anterior. }\end{array}$ & $\begin{array}{l}\text { Oportunidade de } \\
\text { melhoria } \\
\text { relevante, pode ser } \\
\text { ignorada. }\end{array}$ \\
\hline e) & Atrativo & Alta & Igual & Melhorar & $\begin{array}{c}\text { Experiência anterior } \\
\text { com } \\
\text { outro serviço, leva o } \\
\text { consumidor a considerar } \\
\text { o atributo importante. }\end{array}$ & $\begin{array}{l}\text { Melhoria de um } \\
\text { atributo não-crítico. }\end{array}$ \\
\hline f) & Atrativo & Alta & Superior & Manter & - & Decisão Correta. \\
\hline g) & Unidimensional & Alta & $\begin{array}{l}\text { Inferior (Ponto } \\
\text { Fraco) }\end{array}$ & Melhorar & - & Decisão Correta. \\
\hline h) & Unidimensional & Alta & $\begin{array}{l}\text { Superior (Ponto } \\
\text { Forte) }\end{array}$ & $\begin{array}{l}\text { Manter ou } \\
\text { Melhorar em } \\
\text { segundo } \\
\text { plano }\end{array}$ & - & Decisão Correta. \\
\hline
\end{tabular}

Fonte: Tontini et al. (2005). 


\section{Análise de Correlação no SPSS}

Este método é empregado para analisar a satisfação dos clientes que pode ser observado nas planilhas por meio do software IBM-SPSS. O objetivo do procedimento é introduzir técnicas que permitam organizar e apresentar os dados, de maneira que possam ser interpretados de acordo com os objetivos da pesquisa. A avaliação dos atributos juntamente com a correlação em que eles apresentam, demonstram sua significância na satisfação média, apontando as melhorias que devem ser estabelecidas nos devidos atributos.

Tabela 5: SPSS - Correlação de Spearmann x Média da satisfação.

\begin{tabular}{|c|c|c|c|c|}
\hline Atributo & Correlação & Significância & Satisfação Média & Ranking Melhoria \\
\hline Atributos abaixo da média & & & & \\
\hline Prontidão dos Funcionários &, $627^{* *}$ & Correlação substancial & 3,11 & $1^{\circ}$ lugar \\
\hline Rapidez na medição &, $547^{* *}$ & Correlação substancial & 2,96 & $2^{\circ}$ lugar \\
\hline Acessibilidade com donos &, $580^{* *}$ & Correlação substancial & 2,98 & $3^{\circ}$ Iugar \\
\hline Demora na entrega &, $400^{* *}$ & Correlação substancial & 2,18 & $4^{\circ}$ Iugar \\
\hline Atributos acima da média & & & & $\mathrm{A}$ \\
\hline Apresentação dos funcionários &, 104 & Correlação fraca & 3,62 & $\mathrm{~B}$ \\
\hline Rapidez no atendimento &, $179^{*}$ & Correlação fraca & 3,31 & $\mathrm{C}$ \\
\hline Gentileza no atendimento &, $378^{* *}$ & Correlação fraca & 3,11 & $\mathrm{D}$ \\
\hline Conservação dos vidros &, $394^{* *}$ & Correlação fraca & 3,25 & $\mathrm{E}$ \\
\hline Atendimento por telefone &, $401^{* *}$ & Correlação substancial & 3,03 & $\mathrm{~F}$ \\
\hline Variedade de produtos &, $460^{* *}$ & Correlação substancial & 3,34 & $\mathrm{G}$ \\
\hline Opções de pagamento &, $494^{* *}$ & Correlação substancial & 3,13 & $\mathrm{H}$ \\
\hline Atendimento em domicílio &, $550^{* *}$ & Correlação substancial & 3,11 & $\mathrm{I}$ \\
\hline Preços &, $583^{* *}$ & Correlação substancial & 3,19 & \\
\hline Média da satisfação geral & 1,000 & & 3,01 & \\
\hline
\end{tabular}

Conforme avaliação da tabela SPSS, caracterizou-se os atributos que ficaram acima da média sendo eles: (A) Apresentação dos funcionários, (B) Rapidez no atendimento, (C) Gentileza no atendimento, (D) Conservação dos vidros tiveram significância de correlação fraca. (E) Atendimento por telefone, (F) Variedade de produtos, (G) Opções de pagamento, (H) Atendimento em domicílio, (I) Preços, tiveram significância de correlação substancial comparadas aos demais atributos.

Para os demais atributos que ficaram abaixo da média classificaram-se em: 10 lugar, prontidão dos funcionários, $2^{\circ}$ lugar, Rapidez na medição, $3^{\circ}$ lugar acessibilidade com os donos, $4^{\circ}$ demora na entrega. Todos tiveram significância de correlação substancial. Através da coleta destes dados se pode identificar que os pontos mais importantes foram, as correlações substanciais, pois atingiram pontuação mais elevada, demostrando assim sua importância para a Vidraçaria União.

\section{Pontos fortes, pontos fracos e sugestões de melhoria}

\section{Pontos fortes}

Dos 134 respondentes, cerca de 31,5\% responderam a esta questão. Os dados obtidos são apresentados na tabela 6 . Percebe-se uma grande satisfação dos clientes no que diz respeito à conservação

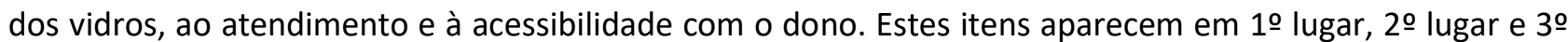
lugar respectivamente demonstrando os pontos fortes mais citados pelos respondentes. Outros Pontos fortes se demonstram importantes como Gentileza, Qualidade dos produtos, Rapidez no atendimento do 
caixa e Preços acessíveis, sendo mencionados 14 vezes, 11 vezes, 11 vezes e 11 vezes respectivamente.

Tabela 6: Pontos fortes indicados.

\begin{tabular}{|l|c|l|c|}
\hline \multicolumn{1}{|c|}{ Pontos fortes } & Citações & \multicolumn{1}{c|}{ Pontos fortes } & Citações \\
\hline Conservação dos Vidros & 27 & Preços Acessíveis & 11 \\
\hline Atendimento & 21 & Variedades & 9 \\
\hline Acessibilidade Com o Dono & 19 & Prontidão dos Funcionários & 9 \\
\hline Gentileza & 14 & Rapidez no Atendimento & 7 \\
\hline Qualidade dos Produtos & 11 & Entrega no Prazo & 5 \\
\hline Rapidez No Atendimento do Caixa & 11 & & - \\
\hline
\end{tabular}

\section{Pontos Fracos}

Dos 134 respondentes, cerca de $25 \%$ dos clientes responderam à questão sobre os pontos fracos. Os dados obtidos são apresentados na Tabela 7. É possível verificar, por meio da Tabela 7, que os pontos fracos com maior percepção por parte dos clientes são: Demora na entrega e Opções de pagamento. Outros pontos fracos não são significativos, uma vez que foram pouco percebidos e mencionados pelos clientes. Com relação aos problemas citados pelos clientes é possível realizar uma análise e criar uma estratégia para solucioná-los de maneira mais rápida, pois há a tendência de que eles busquem empresas que se mostrem confiáveis, com excelência em serviços interativos ,além de preparadas para reparar os seus erros e que tenham comportamento ético impecável (BERRY, 1996). Conforme defendido por Berry et al. (1995), a empresa deve identificar todas as decepções do cliente, mesmo que estas sejam pequenas, pois compreendendo o comportamento do consumidor, torna-se possível reparar as frustrações e conseguir mantê-los se relacionando com a empresa.

Tabela 7: Pontos Fracos Indicados.

\begin{tabular}{|l|c|}
\hline \multicolumn{1}{|c|}{ Pontos fracos } & \multicolumn{1}{c|}{ Citações } \\
\hline Demora na Entrega & 12 \\
\hline Opções de Pagamento & 9 \\
\hline Preços & 7 \\
\hline Prontidão dos Funcionários & 3 \\
\hline Variedade de Produtos & 2 \\
\hline
\end{tabular}

\section{Sugestões de melhoria}

As pesquisas de opinião permitem às empresas direcionarem suas ações no mercado, uma vez que se ouve a voz do cliente, é possível direcioná-la para as melhorias dos serviços, mantendo os consumidores atuais e buscando um maior espaço no mercado competitivo. Dos 134 respondentes, obteve-se 39 respostas à questão. Os dados obtidos se encontram na tabela 8.

Tabela 8: Sugestões de melhoria.

\begin{tabular}{|l|c|}
\hline \multicolumn{1}{|c|}{ Sugestões } & Citações \\
\hline Fazer Orçamento Mais Rápido & 10 \\
\hline Fazer Ofertas & 9 \\
\hline Preços Mais Baixos & 8 \\
\hline Maior Variedade de Produtos & 6 \\
\hline Mais Atendentes & 3 \\
\hline Entregar Mais Rápido & 2 \\
\hline Climatização & \\
\hline
\end{tabular}

Por meio da tabela 8, observa-se que os clientes almejam algumas melhorias nos serviços prestados 
pela empresa. As sugestões mais relevantes são: Fazer orçamento mais rápido, citado 10 vezes, fazer ofertas ( 9 vezes) e preços mais baixos (8 vezes).

\section{CONCLUSÕES}

Definiu-se como objetivos deste artigo: A identificação de oportunidades de melhoria com relação a satisfação dos clientes da loja através de análise conjunta da Matriz de Importância x Desempenho e o Modelo Kano de Qualidade Atrativa e Obrigatória e a identificação de atributos de melhoria nos serviços prestados pela empresa.

O presente estudo utilizou-se do modelo Kano com escala adaptada de Tontini (2003) e da Matriz de Importância x Desempenho. No questionário, cada respondente apontou seu grau de satisfação ou insatisfação com cada um dos 13 atributos utilizados, além do grau de importância de cada um. 0 objetivo da pesquisa foi concluído com sucesso, atingindo a quantidade de 134 respondentes. O diagnóstico dos dados obtidos apontou que os pontos de maior desempenho da vidraçaria são: rapidez no atendimento (3), apresentação dos funcionários (4) e Variedade de produtos (8). De maneira geral a empresa mantém seus clientes satisfeitos, porém deve melhorar com relação aos atributos seguintes. Rapidez na medição (6); Acessibilidade com donos (9); demora na entrega (11) e Prontidão dos funcionários (2), para quem sabe chegar aos $100 \%$ de satisfação.

O aprimoramento dos atributos pode levar a uma maior satisfação dos clientes com relação à empresa como um todo. Aumentar a satisfação pode levar à uma maior fidelização de clientes, o que é um efeito de grande relevância para a empresa, assim como a diminuição do desempenho dos atributos pode levar à insatisfação e outros prejuízos para a empresa.

\section{REFERÊNCIAS}

BAUER, F.; MATZLER, K.. Antecedents of M\&A success: The role of strategic complementarity, cultural fit, and degree and speed of integration. Strategic management journal, v.35, n.2, p.269-291, 2014.

BEDDOW, T. A.; VAN LEEUWEN, J. L.; JOHNSTON, I. A.. Swimming kinematics of fast starts are altered by temperature acclimation in the marine fish Myoxocephalus scorpius. Journal of Experimental Biology, v.198, 203-208, 1995.

BERGER, A. N.. Special Issue on The Efficiency of Financial Institutions. Journal of Banking \& Finance, v.17, n.2-3, p.221-249, 1993.

BERRY, L. L.; PARASURAMAN, A.. Serviços de marketing: Competindo através da qualidade. São Paulo: Maltese, 1995.

BERRY, L.. Serviços de satisfação máxima. Rio de Janeiro: Campus, 1996.

CYRNE, C.. A Matriz Importância x Desempenho - Avaliando a Percepção dos Alunos Transferidos para Outras IESs. In: COLÓQUIO INTERNACIONAL SOBRE GESTÃO UNIVERSITÁRIA NA AMÉRICA DO SUL, 11. Anais. Florianópolis, 2011.
GARVER, M. S.. Best practices in identifying customer-driven improvement opportunities. Industrial Marketing Management, v.32, n.6, p.455-466, 2003.

GIANESI, I. G. N.; CORRÊA, H. L.. Administração estratégica de serviços: operações para a satisfação do cliente. 1994.

HUISKONEN, J.; PIRTTILÄ, T.. International journal of production economics. Elsevier, 1998.

KANO, N.. Attractive quality and must-be quality. The Journal of Japanese Society for Quality Control, v.14, p.3948, 1984.

KLEIN, M. V.; SLONGO, L. A.. Identificação do nível de relacionamento entre a Claro Digital e seus clientes corporativos. Dissertação (Mestrado) - Universidade Federal do Rio Grande do Sul, Porto Alegre, 2003.

KOTLER, P.. Administração de marketing. 1998.

KOTLER, P.; ARMSTRONG, G.. Princípios de Marketing. 9 ed. São Paulo: Prentice Hall, 2003. 
LAS CASAS, A. L.. Marketing de Serviços. 6 ed. São Paulo: Atlas, 2012

LAS CASAS, A. L.. Treinamento de vendedores no varejo. Revista de Administração da Universidade de São Paulo, v.32, n.2, 1997.

MARINHO, L. M. C. V.. Métodos de precificação: enfoque nas vidraçarias de médio e pequeno porte do Ceará. Dissertação (Mestrado) - Universidade Federal do Ceará, Fortaleza, 2009.

MATTAR, F. N.. Pesquisa de Marketing: Metodologia e Planejamento. São Paulo: Atlas, 1996.

MATZLER, K.; BAILOM, F.; HINTERHUBER, H. H.; RENZEL, B.; PICHLER, J.. The asymmetric relationship between attributelevel performance and overall customer satisfaction: a reconsideration of the importance-performance analysis.
Industrial Marketing Management, v.33, n.4, p.271-277, 2004.

RIBEIRO, J. L. D.; TINOCO, M. A. C.. Determinantes da satisfação e atributos da qualidade em serviços bancários. Gest. Prod., v.17, n.4, p.775-790, 2010.

ROSSI, C.; SLONGO, L.. Editorial. Revista Administração Contemporânea, Curitiba, v.2, n.1, 1998.

TONTINI, G.. Deployment of customer needs in the QFD using a modified Kano model. Journal of Academy of Business and Economics, v.2, n.1, p.103-116, 2003.

TONTINI, G.; SILVEIRA, A.. Identificação de atributos críticos de sucesso em produtos e serviços: uma alternativa a análise de importância $X$ desempenho. In: BALAS ANNUAL CONFERENCE. Anais. 2005. 